\title{
The role of difference between proton and neutron structure functions and related harmonic oscillator parameter to shells mean square radius in the EMC effect of ${ }^{7} \mathrm{Li},{ }^{27} \mathrm{Al},{ }^{28} \mathrm{Si}$ and ${ }^{63} \mathrm{Cu}$ nuclei
}

\section{F. Zolfagharpour*}

Department of physics, University of Mohaghegh Ardabili, P.O. Box 179, Ardabil, Iran E-mail: zolfagharpour@uma.ac.ir

\section{A. Sepahvand}

Azad University of Touyserkan, Department of Physics, P.O. Box 163, Touyserkan, Iran

E-mail: sepahvandali@yahoo.com

\begin{abstract}
In this investigation the neutron and proton GRV's structure function used to calculate nuclear structure function of ${ }^{7} \mathrm{Li},{ }^{27} \mathrm{Al},{ }^{28} \mathrm{Si}$ and ${ }^{63} \mathrm{Cu}$ nuclei. The neutron and proton number of this nuclei are different, so it seems that this difference can not be neglected in the extraction of EMC ratio for this nuclei. We know that binding nucleons in different shells have different momentum distributions and one can relate these momentum distributions to shells mean square radius. In this investigation we considered these two phenomena to extract the EMC ratio for ${ }^{7} \mathrm{Li},{ }^{27} \mathrm{Al}$, ${ }^{28} \mathrm{Si}$ and ${ }^{63} \mathrm{Cu}$ nuclei. The extracted results show good agreement with the experimental data by sufficient depletion in the binding energy.
\end{abstract}

8th Conference Quark Confinement and the Hadron Spectrum September 1-6 2008

Mainz, Germany

* Speaker. 


\begin{tabular}{|c|c|c|c|}
\hline Shell & ${ }^{27} \mathrm{Al}$ & ${ }^{28} \mathrm{Si}$ & ${ }^{63} \mathrm{Cu}$ \\
\hline $0 s$ & $(2,2,-15)(2,2,-2)$ & $2,2,-20)$ & $(2,2,-30)$ \\
\hline $0 p$ & $(1,2,-15)(6,6,-2$ & $(6,6,-20)$ & $(6,6,-25)$ \\
\hline $0 d$ & $(5,6,-1$ & $(6,6,-18)$ & $(10,10,-25)$ \\
\hline $1 s$ & & & $(2,2,-20)$ \\
\hline $0 f$ & & & $(9,10,-20)$ \\
\hline $1 p$ & & & $(0,4,-15)$ \\
\hline
\end{tabular}

Table 1: The brackets contain $\left(g_{n l}^{\mathrm{p}}, g_{n l}^{\mathrm{n}}, \varepsilon_{n l}(M e V)\right)$ for related shell.

\section{Theoretical formalism and conclusion}

One can obtain the nuclear structure function of nucleus by main equation [1]

$$
F_{2}^{A}(x)=\sum_{N=\mathrm{n}, \mathrm{p}} \sum_{n l} \int_{x}^{\infty} d z g_{n l}^{N} f^{N}(z)_{n l} F_{2}^{N}(x / z)
$$

where the first sum is over the proton and neutron cases. The second sum is over the quantum number of each energy levels. The $g_{n l}^{N}$ is the occupation number of energy level $\varepsilon_{n l}$ for proton $(\mathrm{N}=\mathrm{p})$ and neutron $(\mathrm{N}=\mathrm{n}) . F_{2}^{N}(x)$ is the nucleons structure function and we used the GRV's proton and neutron structure functions [2]. For the nucleons distribution and harmonic oscillator parameter we have [1]:

$$
\begin{aligned}
f^{N}(z)_{n l}= & \frac{1}{2}\left(\frac{m_{N}}{\hbar \omega}\right)^{1 / 2} \frac{n !}{\Gamma\left(n+l+\frac{3}{2}\right)} \sum_{t_{1}=0}^{n} \sum_{t_{2}=0}^{n} \frac{(-1)^{t_{1}+t_{2}}}{t_{1} ! t_{2} !}\left(\begin{array}{c}
n+l+\frac{1}{2} \\
n-t_{1}
\end{array}\right) \\
& \times\left(\begin{array}{c}
n+l+\frac{1}{2} \\
n-t_{2}
\end{array}\right) \Gamma\left[l+t_{1}+t_{2}+1, \frac{m_{N}}{\hbar \omega}\left(z-1-\frac{\varepsilon_{n l}}{m_{N}}\right)^{2}\right] .
\end{aligned}
$$

In the natural unit we have [3]:

$$
\hbar \omega=\frac{41.33}{\left.<r^{2}\right\rangle_{n l}}\left(2 n+l+\frac{3}{2}\right) .
$$

The extracted EMC ratio of nuclei ${ }^{7} \mathrm{Li},{ }^{27} \mathrm{Al},{ }^{28} \mathrm{Si}$ and ${ }^{63} \mathrm{Cu}$ shows good agreement with the experimental data. The used binding energy can be found in the table 1 . The results show the isoscaler symmetry breaking and considering different harmonic oscillator parameter for differen shells could get sufficient depletion in the binding energy.

\section{References}

[1] S. V. Akulinichev, et al., Phys. Rev. Lett. 21 (1985) 2239.

[2] M. Glück, E. Reya, A. Vogt, Z. Phys. C 67 (1995) 433.

[3] F. Zolfagharpour, ArXiv: nucl-th/0802.1623. 